\title{
A Morpho-Syntactically Informed LSTM-CRF Model for Named Entity Recognition
}

\author{
Lilia Simeonova \\ FMI \\ Sofia University \\ Bulgaria \\ lilia.valentinova. \\ simeonova \\ @gmail.com
}

\author{
Kiril Simov, Petya Osenova \\ LMaKP \\ IICT-BAS \\ Bulgaria \\ $\{$ kivs, petya \\ abultreebank.org
}

\author{
Preslav Nakov \\ Qatar Computing \\ Research Institute, HBKU \\ Qatar \\ pnakov@qf .org.qa
}

\begin{abstract}
We propose a morphologically informed model for named entity recognition, which is based on LSTM-CRF architecture and combines word embeddings, Bi-LSTM character embeddings, part-ofspeech (POS) tags, and morphological information. While previous work has focused on learning from raw word input, using word and character embeddings only, we show that for morphologically rich languages, such as Bulgarian, access to POS information contributes more to the performance gains than the detailed morphological information. Thus, we show that named entity recognition needs only coarse-grained POS tags, but at the same time it can benefit from simultaneously using some POS information of different granularity. Our evaluation results over a standard dataset show sizeable improvements over the state-of-the-art for Bulgarian NER.
\end{abstract}

\section{Introduction}

Although in recent years the Named Entity Linking (also known as Named Entity Disambiguation) task has been central in NLP research, the Namedentity recognition (NER) task has remained far from solve, having in mind the productivity of names and the amount of information available in the era of big and noisy data.

NER plays a critical role in the processing of texts with application to many real-world Natural Language Processing (NLP) tasks such as Question Answering, Information Extraction, Machine Translation, Dialog Systems, and chatbots, where it is sometimes called Concept Segmentation and Labeling (Saleh et al., 2014).
Traditionally, NER has focused on recognizing entities such as person (PER), organization (ORG), location (LOC), and miscellaneous (MISC). This tradition goes back to the Message Understanding Conference (MUC) for English (Grishman and Sundheim, 1996), and the subsequent CoNLL 2002/2003 shared tasks, which also targeted other European Languages such as Spanish, Dutch, and German (Tjong Kim Sang and De Meulder, 2003). ${ }^{1}$ This same setup was followed in more recent work for a number of other languages, and we also follow it in the present work.

Early systems relied on hand-crafted rules with pattern-matching (Appelt et al., 1995). Unfortunately, this required an large pre-annotated datasets, collecting which was time-consuming and error-prone. The next step was to add gazetteers and lexicons that were generated automatically or semi-automatically (Popescu and Etzioni, 2005). Adding such resources required special approaches to resolve the ambiguity between names and common words. Such problems were solved using models such as Hidden Markov Models (Zhou and Su, 2002) and Conditional Random Fields (Sutton and McCallum, 2012).

In our work here, we use deep neural networks for Bulgarian NER. Lample et al. (2016) have shown remarkable results for English, using a combination of Bi-LSTMs (Bi-directional Long Short-Term Memory) and CRF. However, the approach is problematic for morphologically rich languages. The main problem is the missing information within word embeddings for the numerous word forms involved in multiword names that require additional grammatical knowledge in order to be processed properly. Here we incorporate such information as additional input to our neural model.

\footnotetext{
${ }^{1}$ Other schemata such as ACE (Doddington et al., 2004) used a richer inventory of entity types.
} 
Our contributions are as follows:

- We show that for morphologically rich languages such as Bulgarian the access to POS and morphological annotation is crucial and can yield very sizeable performance gains.

- We achieve sizable improvements over the state-of-the-art for Bulgarian NER.

- Finally, we make our data and code freely available, which should enable direct comparison in future work. ${ }^{2}$

\section{Related Work}

Our work is based on Bulgarian, but we claim that it is appropriate also for other languages with rich morphological systems like Slavic and Romance languages, for example. For that reason, we present first the best results for NER in other Slavic languages having in mind that they are synthetic, while Bulgarian is a predominantly analytic language whose morphological richness lies exclusively in the verbal system and not so much in the nominal one. Analytism implies more types of multiword named entities in Bulgarian but less inflection variety, and different distribution of the common types for these languages. The direct comparison of the numbers presented below should be taken with a grain of salt as they are on different datasets and for different languages. Yet, they are indicative for the different methods used for these languages.

For Russian, a Hybrid Bi-LSTM approach was applied by Le et al. (2018), who achieved precision of 89.57 , recall of 84.89 , and F1 score of 87.17. These results are comparable to the ones by our model using the same approach.

For Czech, Straková et al. (2013) reported precision of 88.27 , recall of 78.00 , and F1 score of 82.82 using a Maximum Entropy Markov Model. The feature modeling also proved to be working in Czech, as their best results used features based on morphological analysis, two-stage prediction, word clustering, and gazetteers.

For Polish, Piskorski et al. (2004) achieved precision of 91.0, recall of 77.5, and F1 score of 82.4. They used the SProUT system, which is an NLP platform, consisting of pattern/action rules.

\footnotetext{
${ }^{2}$ http://github.com/lilia-simeonova/ NER-bg/
}

In the last years, the interest in NER for Slavic languages grew. Two shared tasks were organized - the first and the second Multilingual Named Entity Challenge in Slavic Languages. They have been descibed in (Piskorski et al., 2017) and (Piskorski et al., 2019). The challenges included several tasks: recognition of mentions of named entities in Web documents in seven Slavic languages (Bulgarian, Croatian, Czech, Polish, Russian, Slovak, Slovene, Ukrainian), their normalization/lemmatization as well as cross-lingual linking.

Our evaluation on NER in this paper is more similar to the relaxed evaluation parameter where the string is detected and classified, not the invariant. Considering the complexity of the task, the drop of the results per language and per entity types have been expected. Such a task, however, is also good motivation for improving the NER systems for Slavic languages, including Bulgarian.

There is some previous work on NER for Bulgarian. Georgiev et al. (2009) presented a model using Conditional Random Fields with several hand-crafted features. They combined wellestablished features used for other languages with language-specific lexical, syntactic, and morphological information. Their result is the previous state-of-the-art for Bulgarian.

So far, the highest reported results for NER are for English. For example, Chiu and Nichols (2016) reported an F1 score of 91.20 using BiLSTM + CNN + gazetteers + linking, while Passos et al. (2014) achieved an F1 score of 90.90 using a new form of learning word embeddings that can leverage information from relevant lexicons. For German, Gillick et al. (2016) achieved an F1 score of 82.84 , which shows that the rich morphology causes a drop in the performance.

Currently, the prevalent paradigm in NLP is to use neural networks, typically based on LSTMs or CNNs. As we have mentioned above, Lample et al. (2016) proposed an LSTM-CRF model for NER. ${ }^{3}$ The model uses a bi-directional LSTM to encode the left and the right context of the current input word. Then it passes the concatenation of the two hidden vectors (one produced by the left LSTM and one by the right LSTM) to a CRF model. Its task is to ensure the global consistency of the NER tags.

\footnotetext{
${ }^{3}$ They also proposed a transition-based model inspired by shift-reduce parsers, but the results were worse.
} 
In this model, each input word is represented as a concatenation of its word embedding and the character-level embedding for the word produced by a character Bi-LSTM. The character embedding provides features for the suffix and the prefix of the word. Thus, the left-to-right characterbased LSTM embedding models the word suffix, while the right-to-left one models the word prefix. The word embeddings are trained in an unsupervised manner on external data, while the characterbased LSTM embeddings are trained on the training data as part of the end-to-end training of the full LSTM-CRF model. This model does not need any explicit feature engineering nor does it need manual gazetteers; yet, it achieved state-of-the-art performance for four languages: English, German, Dutch, and Spanish. Here we take this model as a basis, and we augment it to model part-of-speech (POS) and grammatical information, which turns out to be very important for a morphologically complex language such as Bulgarian.

Strubell et al. (2017) extended the above model by substituting the LSTM with Iterated Dilated Convolutional Neural Networks, a variant of $\mathrm{CNN}$, which permit fixed-depth convolutions to run in parallel across entire documents, thus making use of GPUs, which yields up to 20-fold speed up, while retaining performance comparable to that of the LSTM-CRF model. They further aggregated context from the entire input document, which they found to be helpful. In our preliminary monolingual experiments, this model performed very similarly, but slightly worse, than the LSTMCRF model, and thus we chose LSTM-CRF for our experiments below.

\section{Data}

In this paper, we work with a Bulgarian corpus, annotated with BIO tags and positional tags, the same as in the CoNLL-2002 shared task (Tjong Kim Sang, 2002). The data is in BIO format, which encodes for each token in the text whether it is at the beginning of the expression of interest (Named Entity in our case), inside or outside of it. The annotation in the available Bulgarian data comes from the manually annotated Bulgarian treebank, known as BulTreeBank (Simov et al., 2004a). In the treebank, each NE is represented as a constituent consisting of one or more tokens. Each NE phrase is annotated by the categories Person, Organization, Location, and Other.

\begin{tabular}{lllll}
\hline $\begin{array}{l}\text { Xристо } \\
\text { Hristo }\end{array}$ & $\begin{array}{l}\text { Стоичков } \\
\text { Stoichkov }\end{array}$ & $\begin{array}{l}\text { пристигна } \\
\text { arrived }\end{array}$ & $\begin{array}{l}\text { в } \\
\text { in }\end{array}$ & $\begin{array}{l}\text { Coфия } \\
\text { Sofia }\end{array}$ \\
\hline B-PER & I-PER & O & O & B-LOC \\
\hline
\end{tabular}

Table 1: An example in BIO encoding.

The BIO tags for the tokens forming a given named entity (NE) in the treebank are created on the basis of the syntactic annotation. The first word in the phrase is marked as the beginning of the NE, while the rest of the tokens ar emarked as inside of the NE. The tokens that are not part of any NE are encoded as outside elements. In order to represent the category of the NE, each tag for begin and inside tokens includes a modifier for the category. Thus, we use nine labels: B-PER, IPER, B-ORG, I-ORG, B-LOC, I-LOC, B-MISC, I-MISC, O. The example in Table 1 shows a simple sentence annotated with two named entities: a person name (Hristo Stoichkov) and a location name (Sofia).

Besides the BIO tagging, the texts in the dataset inherited the morphosyntactic annotation from the treebank. This annotation uses the BulTreeBank Morphosyntactic Tagset (Simov et al., 2004b). The tagset is positional. It encodes parts-of-speech and grammatical features for Bulgarian. For example, Npfsi stands for noun, proper, feminine, singular, indefinite. This annotation offers an opportunity to explore how the morphological features can affect NER.

The resulting dataset is divided into three disjoint sets: training set (Train), development set (Dev), and test set (Test). Table 2 shows statistics about the annotated data. We can see that a large number of examples are labeled as Person names, and that the distribution of Locations, Persons and Organizations is not balanced. While we can still build a stable system based on this data, the class imbalance makes our model more vulnerable to overfitting. Thus, we use early stopping in order to prevent the model from continuing to learn weights and parameters if it does not see an improvement in the final score.

\begin{tabular}{lrrrrrr}
\hline & Sent. & Tokens & PER & ORG & LOC & MISC \\
\hline Train & 28,636 & 528,567 & 16,804 & 3,028 & 6,786 & 911 \\
Dev & 4,063 & 64,014 & 2,514 & 515 & 1,021 & 227 \\
Test & 3,907 & 60,645 & 1,875 & 305 & 781 & 112 \\
\hline
\end{tabular}

Table 2: Statistics about the data. 


\section{Model}

As mentioned above, we construct our model as a modification of the Bi-LSTM-CRF architecture from (Lample et al., 2016). After some experiments with the original system, we decided to modify its input: we added a vector representing some of the information encoded in the morphosyntactic tags. Thus, we created the input vectors for the tokens in the sentences as a concatenation of three vectors: a word embedding vector, a character embedding vector, and a vector containing some grammatical features, called a grammatical vector. We experimented with different grammatical vectors, as explained below.

The rest of the Bi-LSTM-CRF architecture of (Lample et al., 2016) was kept as in the original model: First, we run a Bi-LSTM over the sequence of word vectors so that we could get their contextual word representation. We use a fully connected neural network to get a score for each of the tags. At the end, we run a CRF decoder to decide what the best combination of scores is.

The key takeout of our model is that we use some feature modeling to show that for a morphologically rich language such as Bulgarian using POS and grammatical information can improve the results. Thus, we mix automatically learned features - the word and the character embeddings - , with hand-crafted features encoded as a grammatical vector.

In the rest of this section, we describe the different components of our system.

LSTM-CRF Implementation For the implementation of the general LSTM-CRF architecture, we use Tensorflow (Sak et al., 2014).

Word Embedding Nowadays there are many different approaches to train word vectors such as Word2vec (Mikolov et al., 2013), GloVe (Pennington et al., 2014), FastText (Bojanowski et al., 2017), and many more. In our experiments, we use the pre-trained Bulgarian word embeddings from FastText (Bojanowski et al., 2017). ${ }^{4}$ This choice was motivated by the fact that FastText uses the structure of the words by taking into consideration character $n$-grams, thus modeling morphology and many out-of-vocabulary words.

\footnotetext{
${ }^{4}$ In this work, we do not use any contextualization of the word embeddings such as ElMo (Peters et al., 2018) and BERT (Devlin et al., 2019), as our Bi-LSTM architecture already performs contextualization.
}

Character Bi-LSTM Embedding In order to produce character embeddings, we use a bidirectional LSTM over the character representation of the text. For each character in the text, each of the two LSTMs produces an hidden vector. For each word, the hidden vector for the last character produced by the left-to-right LSTM models information about the suffix of the word. Similarly, the hidden vector for the first character produced by the right-to-left LSTM models information about the prefix of the word. Following the approach, used in (Ling et al., 2015), we constructed the final character embedding of the word as a concatenation of the prefix and the suffix vectors.

Grammatical Vectors We use several types of grammatical vectors or their combinations. They are divided into POS vectors that encode different combinations of parts-of-speech and morphological vectors encoding other grammatical features.

POS Vectors The part-of-speech information for each word is represented as an a one-hot vector with eleven positions. This vector is concatenated to the vector for the word embedding. In the tagset, we have the following parts-of-speech: $\mathbf{N}$ - noun, $\mathbf{A}$ - adjective, $\mathbf{V}$ - verb, $\mathbf{H}$ - hybrid, $\mathbf{D}$ - adverb, $\mathbf{R}$ - preposition, $\mathbf{P}$ - pronoun, $\mathbf{C}-$ conjunction, $\mathbf{T}-$ particle, $\mathbf{M}$ - numeral, and $\mathbf{I}$ - interjection. In our experiments, we divided these parts-of-speech into different groups depending on the role they play in the representation of the named entities. For example, the tags $\mathrm{A}, \mathrm{N}, \mathrm{H}, \mathrm{R}$ were viewed as a possible part of a named entity in contrast to the others that cannot form named entities. In this case, the one-hot vector contains only two positions. The groups are given in the experimental section below. The Hybrid tag $(\mathrm{H})$ is special in the tagset. It refers to both family names and name adjectives. Bulgarian family names (as other Slavic ones) are proper names, but morphologically they behave like adjectives due to their adjectival origin.

Morphological Vectors The nominal system of Bulgarian shares some features with other Slavic languages, such as agreement in grammatical gender and number, rich pronoun system, etc. However, it has also specific features, such as the postpositioned definite article and lost nominal declension system. Our aim is to show the contribution of all these types of features to the named entity recognition task. 


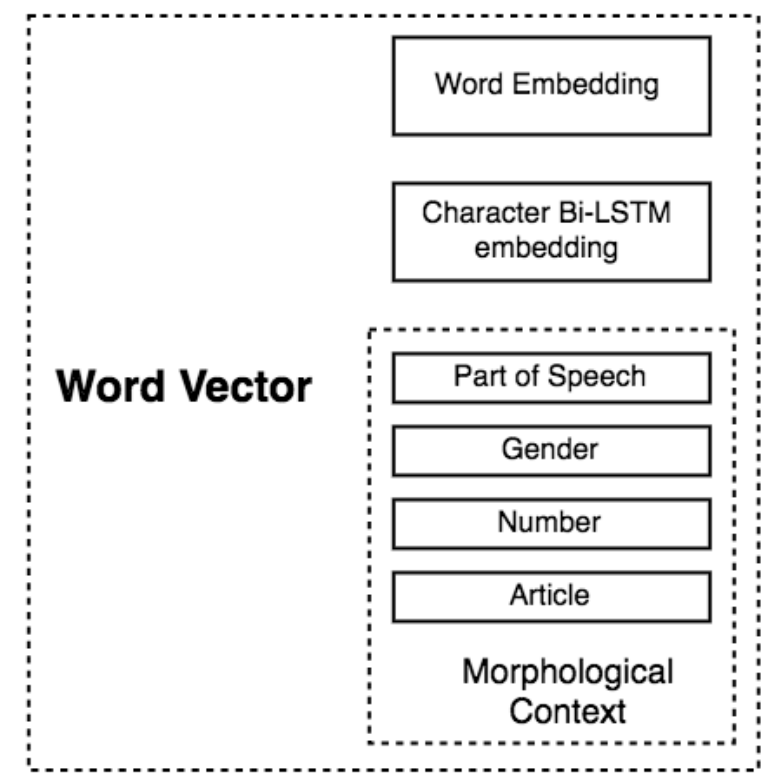

Figure 1: Full input vector representation with concatenation of word embeddings, character embeddings, and morphological features.

Another thing worth mentioning is that the nouns, adjectives and hybrid tags share some common features. This information appears to be very useful for recognizing the more specific types of named entities. Note that the existence of a preposition in a sequence can distinguish some further patterns as well.

The morphological features vary between the different entities, but there are few that can be defined for nouns, adjectives, hybrid tags and pronouns. Some of them are arguably useful, such as gender, number, and definiteness, and we will describe them briefly here:

Gender can have three values: masculine, feminine, and neutral;

Number can have four values: singular, plural, only plural and count form (which is only for masculine nouns for non-persons)'

Definiteness can have four values: indefinite, definite, short definite and full definite. The latter two are for singular masculine nouns only.

For each word with a POS tag of noun, pronoun, adjective or hybrid, we concatenate a one-hot vector representation for each of the features above (we use a zero vector for the rest). We form the final version of our word vector as a concatenation of all contextual vectors, as shown on Figure 1.
Dropout In order to prevent overfitting, we use a dropout layer on top of our word vectors as described in (Hinton et al., 2012). For each presentation of each training example, we randomly exclude a hidden unit from the network with a certain probability. In this way, the system learns to detect and use more useful features.

\section{Experiments and Evaluation}

In this section, we present the experimental setup and the evaluation results for the different models we experimented with.

\subsection{Training and Hyper-parameters}

We experimented with different values of the hyper-parameters and we found that changing some of them can result in sizeable improvements. The most considerable difference was for the learning method and the learning rate. Our best resulst were achieved using the Adam optimizer (Kingma and $\mathrm{Ba}, 2014$ ), which is computationally efficient and has minimal memory requirements.

At the beginning, we set the learning rate to the initial value of 0.001 , and then at each epoch, we multiplied it by a specific learning decay value. Decreasing our learning rate over time can help us find the minimum of our function without actually missing it. While Adam already decays the learning rate at each iteration, previous work has found that tuning the initial learning rate could yield sizeable improvements over the default settings. Thus, we use this additional decay. (Wilson et al., 2017)

The word embeddings we use from FastText have a dimensionality of 300 , while the character embedding vectors have a dimensionality of 100 . In order to produce them, we uses the TensorFlow default Xavier initializer and then we ran a BiLSTM on top of them in order to obtain contextual vectors with no additional layers.

We set the batch size to 20 and the dropout to 2. The Adam's parameters we used are as follows: learningrate $=0.001 ; \beta 1=0.9 ; \beta 2=0.999$; $\epsilon=1 e-08$; use_locking $=$ False. We also added a gradient clipping with a value of 1 .

At decoding time, we used a linear-chain CRF (Lafferty, 2001). This model has been shown to outperform a simple SoftMax classifier as the tagging decision needs to be global. 


\begin{tabular}{lcccc}
\hline \multirow{2}{*}{ Model } & No Morphology & \multicolumn{3}{c}{ With Morpholofy } \\
& F1 & F1 & P & R \\
\hline Model + POS-2 & 90.04 & 90.44 & 92.02 & 88.90 \\
Model + POS-3 & 91.20 & 91.11 & 91.66 & 90.57 \\
Model + POS-4 & 90.16 & 90.83 & 92.30 & 89.41 \\
Model + POS-5 & 91.32 & 90.58 & 92.42 & 88.82 \\
Model + POS-11 & 90.96 & 91.03 & 91.60 & 90.48 \\
\hline Model + POS-3+11 & 91.18 & 92.20 & 93.31 & 91.12 \\
Model + POS-4+11 & 90.89 & 91.04 & 92.17 & 89.94 \\
\hline
\end{tabular}

Table 3: Evaluation results for Bulgarian POS tagging. Shown are results where the standard input to the Bi-LSTM-CRF model is augmented with different POS tags and morphological features.

\begin{tabular}{llllllll}
\hline $\begin{array}{l}\text { English } \\
\text { Bulgarian }\end{array}$ & \multicolumn{6}{c}{$\begin{array}{l}\text { Ivan Valtchev visited the Bulgarian Academy of Sciences. } \\
\text { Иван Вълчев посети Българската академия на науките. }\end{array}$} \\
\hline Tokens & Иван & Вълчев & посети & Българската & академия & на & науките \\
\hline POS11 & N & H & V & A & N & R & N \\
POS2 & ANHR & ANHR & O & ANHR & ANHR & ANHR & ANHR \\
POS3 & ANH & ANH & O & ANH & ANH & R & ANH \\
POS4 & NH & NH & O & A & NH & R & NH \\
POS5 & N & H & O & A & N & R & N \\
\hline
\end{tabular}

Table 4: Example of the POS tags for annotation schemes of different granularities when applied to the same Bulgarian sentence.

\subsection{Experiments}

The experimental results suggest that adding grammatical features can have a sizeable impact on the performance of the general LSTM-CRF model for Bulgarian NER. In Table 3, we can see an example of different combinations of POS tags, where Model stands for Bi-LSTM-CRF and POS represents a one-hot encoding for the following:

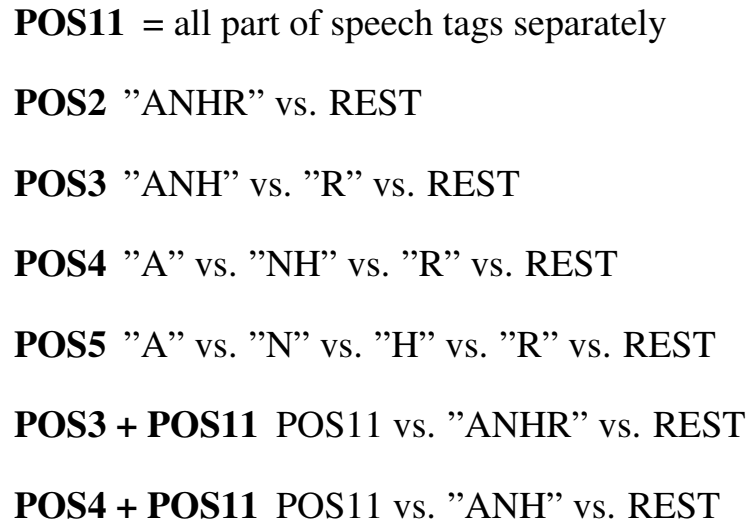

We further perform several experiments in order to determine whether we need the full set of partof-speech tags or it is enough just to know whether the entity is part of the group of the nouns, adjectives, hybrid tags, and prepositions.

\begin{tabular}{ll}
\hline Model & F1 \\
\hline (Georgiev et al., 2009) & 89.40 \\
Our model & 92.20 \\
\hline
\end{tabular}

Table 5: Comparing the previous state-of-the-art results to our best morphologically informed $\mathrm{Bi}$ LSTM-CRF model.

In Table 4, we see how the entities map to the different POS groups. It appears that knowing the concrete tag of each entity can help us improve the performance by almost one percent.

Table 5 shows our best result compared to the previous state-of-the-art result as reported in (Georgiev et al., 2009). They achieved an F1 score of $89.4 \%$ by sing regular expressions, gazetteers and non-local morpho-syntactic characteristics. Our model improves this to $92.20 \%$ without using any external resources.

More detailed evaluation results for our best model are presented in Table 6, where we show the precision, recall and F1 score for each type of named entity. We can observe relatively worse F1 score of 84.70 for Organization compared to 95.86 for Location and 94.95 for Person. We explain this drop in F1 score by the fact that many organizations are named after persons. 


\begin{tabular}{llll}
\hline Entity & Precision & Recall & F1 \\
\hline Location & 97.75 & 94.05 & 95.86 \\
Person & 95.67 & 94.23 & 94.95 \\
Organization & 75.57 & 96.34 & 84.70 \\
Miscellaneous & 96.15 & 22.73 & 36.76 \\
\hline Overall & 93.31 & 91.12 & 92.20 \\
\hline
\end{tabular}

Table 6: Detailed results for the different kinds of named entities.

\begin{tabular}{lc}
\hline Model & F1 \\
\hline (1) LSTM-CRF (words only) & 82.03 \\
(2) fwd-LSTM-char + (1) & 85.15 \\
(3) bwd-LSTM-char + (1) & 85.40 \\
(4) Bi-LSTM-char + (1) & 86.44 \\
(5) POS11 + (4) & 90.96 \\
(6) Morph + (5) & 91.03 \\
(7) POS3 + (6) & 92.20 \\
\hline
\end{tabular}

Table 7: The impact of different components and different component combinations on the performance of our best model.

Table 7 shows the cumulative effect of adding different components to our model. The basic model we started with is shown on line (4). Then, on lines (1)-(3) we remove different components from this basic model, and on lines (5)-(7) we add POS and morphological information to it. We can see sizeable improvement for the standard BiLSTM-CRF model with only word vector representationa and the model with character-level LSTM. Interestingly, there is almost no difference between the suffix and the prefix vectors. We can further see that adding POS11 (5) vector improves the performance by almost four percent absolute. The morphological vectors and POS3 also improved the F1 score to 92.20 points absolute. These improvements show that using known linguistic knowledge such as grammatical features could improve the representation vectors learned over huge text corpora. From the point of view of feature learning, we speculate that vectors trained over texts in morphologically rich languages do not learn enough grammar such as POS and morphology. The character embeddings also seem not to help much. One explanation for this could be that the suffixes and prefixes in Bulgarian are also highly ambiguous.

\begin{tabular}{ll}
\hline Model & Word (Bulgarian / English) \\
\hline Model & Еминем / Eminem \\
Model + POS11 & Фердинанд / Ferdinand \\
Model + POS2 & Ваксберг / Vaksberg \\
Model + POS3 & Гьоте / Goethe \\
Model + POS4 & Обзървър / Observer \\
Model + POS5 & Шехеразада / Scheherazade \\
\hline Model + POS11 + POS3 & Ингмар / Ingmar \\
\hline
\end{tabular}

Table 8: Examples of words which could not be handled correctly by the specific configuration

\section{Error Analysis}

Our manual analysis of the errors shows that one of the main reasons for our model to work better when POS tags are provided is due to the presence of many loanwords in the Bulgarian text. The LSTM-CRF model manages to learn the grammar of the language itself, but it needs additional help with words borrowed from other languages.

A common problem for the LSTM-CRF model is the mislabeling of foreign person or organization names. In such cases, the POS tags help by suggesting the possible part-of-speech for each word. In our test set, around $10 \%$ of the wrongly labeled words are loan words, borrowed primarily from English, Russian, German and Turkish. Table 8 , shows some examples of words that could not be handled properly.

The loanwords cannot be successfully recognized by our algorithm in all cases. Even though some people try to write them in Cyrillic, their structure is different from the typical structure of the Bulgarian words. That is why, we further tried to add gazetteers and lexicons with existing loan words in Bulgarian. Similarly to the way we added POS vectors, we created a one-hot vector for each word that says whether that word is part of our lexicon with loan words or not. We then concatenated the vector to the rest of the word embeddings. However, this approach did not result in significant improvements because new words are added to the language almost every day, and it is impossible to capture them all.

Sometimes, the loanwords come in the Latin alphabet, as they are spelled in their original language. For such cases, we added a feature to the model that captures the information whether the words are in Latin or in Cyrillic. This feature, by itself, did not make much of a difference. Yet, we plan to explore it further in our future work. 


\begin{tabular}{rrrrrrrrrr}
\hline & B-ORG & I-ORG & B-PER & I-PER & B-LOC & I-LOC & B-MISC & I-MISC & O \\
\hline B-ORG & $\mathbf{2 5 8}$ & 0 & 1 & 0 & 2 & 0 & 6 & 0 & 6 \\
I-ORG & 0 & $\mathbf{3 1}$ & 0 & 0 & 0 & 0 & 0 & 0 & 1 \\
B-PER & 9 & 1 & $\mathbf{1 , 1 6 9}$ & 6 & 8 & 0 & 7 & 0 & 58 \\
I-PER & 0 & 1 & 15 & $\mathbf{5 9 5}$ & 0 & 0 & 0 & 0 & 6 \\
B-LOC & 15 & 0 & 8 & 0 & $\mathbf{6 7 6}$ & 0 & 4 & 0 & 36 \\
I-LOC & 1 & 0 & 0 & 1 & 3 & $\mathbf{3 6}$ & 0 & 0 & 1 \\
B-MISC & 27 & 0 & 2 & 0 & 1 & 0 & $\mathbf{3 9}$ & 0 & 41 \\
I-MISC & 0 & 1 & 0 & 0 & 0 & 0 & 0 & $\mathbf{1}$ & 0 \\
O & 12 & 2 & 10 & 22 & 2 & 0 & 3 & 0 & $\mathbf{5 7 , 5 2 2}$ \\
\hline
\end{tabular}

Table 9: Confusion matrix for our best model on the test dataset: the columns represent the true labels and the rows show the predictions.

Table 9 shows a confusion matrix for the nine BIO tags that we used for the four kinds of named entities that we are recognizing. In the table, the columns represent the actual expected gold tags, while the rows show the predictions of our model. There are several interesting observations that we can make about this confusion matrix. First, it looks like the biggest problem for the model is with the tag B-PER, which is often confused with the tag O, i.e., Outside. This is probably due to the fact that in Bulgarian the first names sometimes have more than one meaning, which can confuse the model. The same argument holds for the tag B-LOC, which is also often confused with the tag O. Another place for improvements would be to distinguish better between B-LOC and B-ORG, as many places and organization have identical names, or at least the identical first words. The miscellaneous entities such as the names of books or movies can also have names that are identical to those of some organizations. Even more often, Miscellaneous entities could be confused with the Other category as they contain many common Bulgarian words.

\section{Conclusion and Future Work}

We explored the potential of using morphological information to a recurrent Bi-LSTM-CRF neural network architecture with the aim to improve named entity recognition for morphologically rich languages such as Bulgarian, which pose different challenges for named entity recognition compared to English. Our experiments have shown that adding morphological and part-of-speech information to the model's input yields sizable performance gains over a model that only relies on word-level and character-level embeddings as an input to the neural network.
In future work, we plan to extend the modeling of the morphological structure of the entities. Here, we only used a limited number of features, namely gender, number and definiteness, but it might be interesting to add the full linguistic knowledge encoded in the BulTreebank. We further plan to explore features and models that can help identify loan words in Bulgarian.

Another promising research direction is to compare the differences in the graphical representation of named entities in Bulgarian and English. For example, in English all components of a named entity are capitalized (except for the functional words). In order to have comparable data, we envision to pre-transform the Bulgarian dataset to which to apply the English capitalization rule for the phrasal named entities.

Finally, we plan to experiment with different monolingual representations from ElMo (Peters et al., 2018), BERT (Devlin et al., 2019), ROBERTa (Liu et al., 2019c), XLNet (Yang et al., 2019), and Ernie 2.0 (Sun et al., 2019), pooled representations from Flair (Akbik et al., 2019), distilled representations from MT-DNN (Liu et al., 2019a,b) or cross-language representations from XLM (Lample and Conneau, 2019).

\section{Acknowledgements}

This research was partially supported by the Bulgarian National Interdisciplinary Research eInfrastructure for Resources and Technologies in favor of the Bulgarian Language and Cultural Heritage, part of the EU infrastructures CLARIN and DARIAH - CLaDA-BG, Grant number DO01$164 / 28.08 .2018$

We would like to thank the anonymous reviewers for their constructive comments, which have helped us improve the paper. 


\section{References}

Alan Akbik, Tanja Bergmann, and Roland Vollgraf. 2019. Pooled contextualized embeddings for named entity recognition. In Proceedings of the 2019 Conference of the North American Chapter of the Association for Computational Linguistics: Human Language Technologies. Minneapolis, MN, USA, NAACL-HLT' '19, pages 724-728.

Douglas E. Appelt, Jerry R. Hobbs, John Bear, David Israel, Megumi Kameyama, David Martin, Karen Myers, and Mabry Tyson. 1995. SRI international FASTUS system: MUC-6 test results and analysis. In Proceedings of the 6th Conference on Message Understanding. Columbia, MD, USA, MUC6 '95, pages 237-248.

Piotr Bojanowski, Edouard Grave, Armand Joulin, and Tomas Mikolov. 2017. Enriching word vectors with subword information. Transactions of the Association for Computational Linguistics 5:135-146.

Jason P.C. Chiu and Eric Nichols. 2016. Named entity recognition with bidirectional LSTM-CNNs. Transactions of the Association for Computational Linguistics 4:357-370.

Jacob Devlin, Ming-Wei Chang, Kenton Lee, and Kristina Toutanova. 2019. BERT: Pre-training of deep bidirectional transformers for language understanding. In Proceedings of the 2019 Conference of the North American Chapter of the Association for Computational Linguistics. Minneapolis, MN, USA, NAACL-HLT'2019, pages 4171-4186.

George Doddington, Alexis Mitchell, Mark Przybocki, Lance Ramshaw, Stephanie Strassel, and Ralph Weischedel. 2004. The automatic content extraction (ACE) program - tasks, data, and evaluation. In Proceedings of the Fourth International Conference on Language Resources and Evaluation. Lisbon, Portugal, LREC '04.

Georgi Georgiev, Preslav Nakov, Kuzman Ganchev, Petya Osenova, and Kiril Simov. 2009. Featurerich named entity recognition for Bulgarian using conditional random fields. In Proceedings of the International Conference on Recent Adcances in Natural Language Processing. Borovets, Bulgaria, RANLP'09, pages 113-117.

Dan Gillick, Cliff Brunk, Oriol Vinyals, and Amarnag Subramanya. 2016. Multilingual language processing from bytes. In Proceedings of the 2016 Conference of the North American Chapter of the Association for Computational Linguistics: Human Language Technologies. San Diego, CA, USA, NAACLHLT' 16, pages 1296-1306.

Ralph Grishman and Beth Sundheim. 1996. Message understanding conference-6: A brief history. In Proceedings of the 16th Conference on Computational Linguistics. Copenhagen, Denmark, COLING '96, pages 466-471.
Geoffrey E. Hinton, Nitish Srivastava, Alex Krizhevsky, Ilya Sutskever, and Ruslan Salakhutdinov. 2012. Improving neural networks by preventing co-adaptation of feature detectors. CoRR abs/1207.0580.

Diederik P. Kingma and Jimmy Ba. 2014. Adam: A method for stochastic optimization. CoRR abs/1412.6980.

John Lafferty. 2001. Conditional Random Fields: Probabilistic Models for Segmenting and Labeling Sequence Data. In Proceedings of the 18th International Conference on Machine Learning. Morgan Kaufmann, ICML '01, pages 282-289.

Guillaume Lample, Miguel Ballesteros, Sandeep Subramanian, Kazuya Kawakami, and Chris Dyer. 2016. Neural architectures for named entity recognition. In Proceedings of the 2016 Conference of the North American Chapter of the Association for Computational Linguistics: Human Language Technologies. San Diego, CA, USA, NAACL-HLT'16, pages 260-270.

Guillaume Lample and Alexis Conneau. 2019. Crosslingual language model pretraining. CoRR abs/1901.07291.

The Anh Le, Mikhail Y. Arkhipov, and Mikhail S. Burtsev. 2018. Application of a hybrid Bi-LSTM-CRF model to the task of Russian named entity recognition. In Andrey Filchenkov, Lidia Pivovarova, and Jan Žižka, editors, Artificial Intelligence and Natural Language. Springer International Publishing, pages $91-103$.

Wang Ling, Chris Dyer, Alan W Black, Isabel Trancoso, Ramón Fermandez, Silvio Amir, Luís Marujo, and Tiago Luís. 2015. Finding function in form: Compositional character models for open vocabulary word representation. In Proceedings of the 2015 Conference on Empirical Methods in Natural Language Processing. Lisbon, Portugal, EMNLP' 15, pages $1520-1530$.

Xiaodong Liu, Pengcheng He, Weizhu Chen, and Jianfeng Gao. 2019a. Improving multi-task deep neural networks via knowledge distillation for natural language understanding. CoRR abs/1904.09482.

Xiaodong Liu, Pengcheng He, Weizhu Chen, and Jianfeng Gao. 2019b. Multi-task deep neural networks for natural language understanding. CoRR abs/1901.11504.

Yinhan Liu, Myle Ott, Naman Goyal, Jingfei Du, Mandar Joshi, Danqi Chen, Omer Levy, Mike Lewis, Luke Zettlemoyer, and Veselin Stoyanov. 2019c. RoBERTa: A robustly optimized BERT pretraining approach. CoRR abs/1907.11692.

Tomas Mikolov, Kai Chen, Greg Corrado, and Jeffrey Dean. 2013. Efficient estimation of word representations in vector space. CoRR abs/1301.3781. 
Alexandre Passos, Vineet Kumar, and Andrew McCallum. 2014. Lexicon infused phrase embeddings for named entity resolution. In Proceedings of the Eighteenth Conference on Computational Natural Language Learning. Ann Arbor, MI, USA, CoNLL '14, pages $78-86$.

Jeffrey Pennington, Richard Socher, and Christopher Manning. 2014. GloVe: Global vectors for word representation. In Proceedings of the Conference on Empirical Methods in Natural Language Processing. Doha, Qatar, EMNLP'14, pages 1532-1543.

Matthew Peters, Mark Neumann, Mohit Iyyer, Matt Gardner, Christopher Clark, Kenton Lee, and Luke Zettlemoyer. 2018. Deep contextualized word representations. In Proceedings of the 2018 Conference of the North American Chapter of the Association for Computational Linguistics: Human Language Technologies. New Orleans, LA, USA, NAACLHLT' 18, pages 2227-2237.

Jakub Piskorski, Peter Homola, Małgorzata Marciniak, Agnieszka Mykowiecka, Adam Przepiórkowski, and Marcin Woliński. 2004. Information extraction for Polish using the SProUT platform. In Mieczysław A. Kłopotek, Sławomir T. Wierzchon, and Krzysztof Trojanowski, editors, Intelligent Information Processing and Web Mining. Springer Berlin Heidelberg, pages 227-236.

Jakub Piskorski, Laska Laskova, Michał Marcińczuk, Lidia Pivovarova, Pavel Přibáň, Josef Steinberger, and Roman Yangarber. 2019. The second crosslingual challenge on recognition, normalization, classification, and linking of named entities across Slavic languages. In Proceedings of the 7th Workshop on Balto-Slavic Natural Language Processing. Florence, Italy, BSNLP'19, pages 63-74.

Jakub Piskorski, Lidia Pivovarova, Jan Šnajder, Josef Steinberger, and Roman Yangarber. 2017. The first cross-lingual challenge on recognition, normalization, and matching of named entities in Slavic languages. In Proceedings of the 6th Workshop on Balto-Slavic Natural Language Processing. Valencia, Spain, BSNLP' 17, pages 76-85.

Ana-Maria Popescu and Oren Etzioni. 2005. Extracting product features and opinions from reviews. In Proceedings of the Conference on Human Language Technology and Empirical Methods in Natural Language Processing. Vancouver, Canada, EMNLP '05, pages 339-346.

Hasim Sak, Andrew W. Senior, and Françoise Beaufays. 2014. Long short-term memory based recurrent neural network architectures for large vocabulary speech recognition. CoRR abs/1402.1128.

Iman Saleh, Scott Cyphers, Jim Glass, Shafiq Joty, Lluís Màrquez, Alessandro Moschitti, and Preslav Nakov. 2014. A study of using syntactic and semantic structures for concept segmentation and labeling. In Proceedings of the 25th International
Conference on Computational Linguistics. Dublin, Ireland, COLING '14, pages 193-202.

Kiril Simov, Petya Osenova, Alexander Simov, and Milen Kouylekov. 2004a. Design and implementation of the Bulgarian HPSG-based treebank. In Journal of Research on Language and Computation, Special Issue. Kluwer Academic Publishers, pages 495-522.

Kiril Simov, Petya Osenova, and Milena Slavcheva. 2004b. BTB-TR03: BulTreeBank Morphosyntactic Tagset. BulTreeBank Project, IICT-BAS.

Jana Straková, Milan Straka, and Jan Hajič. 2013. A new state-of-the-art Czech named entity recognizer. In Ivan Habernal and Václav Matoušek, editors, Text, Speech, and Dialogue. Springer Berlin Heidelberg, pages 68-75.

Emma Strubell, Patrick Verga, David Belanger, and Andrew McCallum. 2017. Fast and accurate entity recognition with iterated dilated convolutions. In Proceedings of the Conference on Empirical Methods in Natural Language Processing. Copenhagen, Denmark, EMNLP'17, pages 2670-2680.

Yu Sun, Shuohuan Wang, Yukun Li, Shikun Feng, Hao Tian, Hua Wu, and Haifeng Wang. 2019. ERNIE 2.0: A continual pre-training framework for language understanding. CoRR abs/1907.12412.

Charles Sutton and Andrew McCallum. 2012. An introduction to conditional random fields. Found. Trends Mach. Learn. 4(4):267-373.

Erik F. Tjong Kim Sang. 2002. Introduction to the CoNLL-2002 shared task: Language-independent named entity recognition. In Proceedings of the 6th Conference on Natural Language Learning. Taipei, Taiwan, COLING '02, pages 1-4.

Erik F. Tjong Kim Sang and Fien De Meulder. 2003. Introduction to the CoNLL-2003 shared task: Language-independent named entity recognition. In Proceedings of the Seventh Conference on Natural Language Learning. Edmonton, Canada, CoNLL '03, pages 142-147.

Ashia C. Wilson, Rebecca Roelofs, Mitchell Stern, Nati Srebro, and Benjamin Recht. 2017. The marginal value of adaptive gradient methods in machine learning. In Proceedings of the Conference on Neural Information Processing Systems. Long Beach, CA, USA, NIPS '17, pages 4151-4161.

Zhilin Yang, Zihang Dai, Yiming Yang, Jaime G. Carbonell, Ruslan Salakhutdinov, and Quoc V. Le. 2019. XLNet: Generalized autoregressive pretraining for language understanding. CoRR abs/1906.08237.

GuoDong Zhou and Jian Su. 2002. Named entity recognition using an HMM-based chunk tagger. In Proceedings of the 40th Annual Meeting on Association for Computational Linguistics. Philadelphia, PA, USA, ACL '02, pages 473-480. 